\title{
Transatlantica
}

Revue d'études américaines. American Studies Journal

\section{Pop Life, Art in a Material World.}

Tate Modern, Londres, 1er octobre 2009 - 17 janvier 2010.

\section{Clémence Simon}

\section{(2) OpenEdition}

Journals

Édition électronique

URL : https://journals.openedition.org/transatlantica/4700

DOI : $10.4000 /$ transatlantica. 4700

ISSN : $1765-2766$

Éditeur

Association française d'Etudes Américaines (AFEA)

Référence électronique

Clémence Simon, « Pop Life, Art in a Material World. », Transatlantica [En ligne], 2 | 2009, mis en ligne le 13 novembre 2012, consulté le 01 février 2023. URL : http://journals.openedition.org/transatlantica/ 4700 ; DOI : https://doi.org/10.4000/transatlantica.4700

Ce document a été généré automatiquement le 1 février 2023.

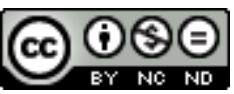

Creative Commons - Attribution - Pas d'Utilisation Commerciale - Pas de Modification 4.0 International - CC BY-NC-ND 4.0

https://creativecommons.org/licenses/by-nc-nd/4.0/ 


\section{Pop Life, Art in a Material World.}

Tate Modern, Londres, 1er octobre 2009 - 17 janvier 2010.

\section{Clémence Simon}

«I don't think myself as evil - just realistic », Andy Warhol ${ }^{1}$

1 «Good business is the best art », légendaire citation d'Andy Warhol, résonne entre les murs de la Tate Modern², digne fil conducteur de l'exposition «Pop Life, Art in a Material World ». Le Business Art et l'artiste intégrant le commercial à son travail (ne se contentant plus de seulement le commenter) réunissent vingt-deux artistes autour de la figure centrale de Warhol, présenté comme le père fondateur de toute cette mouvance pop. L'exposition devait s'appeler « Sold Out ${ }^{3}$ " mais il semblerait que le titre ait déplu à l'un des artistes présents ${ }^{4}$. Cela tombe bien, il est également question d'artiste-star dans « Pop Life » ...

2 Par souci de filiation, c'est naturellement un autoportrait d'Andy Warhol qui accueille le spectateur dès son entrée dans la première des dix-sept salles. Le maître y est entouré de ses deux disciples les plus connus du grand public : à gauche, Koons et son Rabbit ; à droite, Murakami et Hiropon. C'est cette nouvelle Sainte Trinité du Pop qui constituera d'ailleurs l'ossature de l'exposition, constituant les grands repères de l'exposition pendant la visite du spectateur.

3 Dès cette salle introductive, le ton est donné : variété et créativité sont de mise. Le spot de publicité d'Andy Warhol pour TDK ainsi que la vidéo de la parade de Thanksgiving 2007 où le Rabbit de Koons cohabitait avec Snoopy ou encore les figurines shokugan ${ }^{5}$ de Takashi Murakami côtoient sculpture et peinture.

4 La seconde salle prend un caractère soudainement très ludique : consacrée à l'obsession de Warhol pour l'argent, les Gems (1979) ne se dévoilent qu'à la lumière noire. La visite se poursuit au rythme d'une bande son qui est diffusée dans le lointain. La salle 3, Worst of Warhol, présente le format " portrait mondain » qui a permis à Warhol d'approcher le monde des célébrités pour en faire finalement partie. Cette salle marque le plein fonctionnement de la Factory, travaillant « à la chaîne » selon une formule bien rodée : $\$ 25000$ un portrait, un rabais sur le deuxième. L'art devient un véritable commerce. Warhol applique le même traitement à chacun, star comme boîte de soupe. Warhol lui- 
même n'y échappe pas: il se représente sur papier peint (comme il l'avait fait précédemment avec une vache), donnant à sa personne valeur de marque. Il décline son image et celles des icônes warholiennes jusqu'à ce qu'elles sortent de la toile pour intégrer le vocabulaire pictural quotidien. The Pope of Pop est à l'honneur jusqu'à la salle 4, qui sert de transition avec ses « héritiers ": grâce à The Next Step After Art, le spectateur prend plus clairement conscience du développement de la marque Warhol. Cela s'exprime notamment dans la création du magazine Interview en 1969. L'exposition médiatique dont Warhol a été l'objet est ici manifeste : grâce à des clichés de paparazzi ou à ses apparitions télévisées (dans "La croisière s'amuse ", notamment). Une telle médiatisation aurait été difficilement concevable pour un artiste dit sérieux, avant qu'Andy Warhol ne l'intègre à son mode de vie et à son art. A partir de ce moment, les obligations inhérentes au statut d'artiste volent en éclat, lui permettant d'intégrer une sphère plus sociale et mondaine.

5 La filiation avec Warhol n'est jamais loin: en salles cinq et six, Sturtevant utilise le principe de la citation pour reproduire fidèlement (pour leur rendre hommage et les cannibaliser comme l'a fait Warhol ${ }^{6}$ ) des œuvres de Keith Haring... et d'Andy Warhol. Principe également présent chez Richard Prince qui utilise le titre d'une photographie datant de 1923, de Steiglitz, Spiritual America, pour son travail autour de la problématique du statut de star et de la culture de la publicité. Les pièces de Nagy et Vaisman, quand à elles, évoquent la disparition des frontières entre créativité et commerce (les deux artistes étant également galeristes ${ }^{7}$ ).

6 L'une des salles les plus importantes est certainement celle consacrée au Pop Shop de Keith Haring. Une reconstitution du magasin new-yorkais ouvert en 1986 par Haring a été mise en place. Rien ne manque: bande-son rap à plein volume, graphismes entrelacés de l'artiste du sol au plafond, t-shirts et planches de skateboard accrochés aux murs et un vendeur qui attend tout chaland prêt à dégainer sa carte bleue. L'atmosphère y est électrisante, la reconstitution aussi fidèle qu'impressionnante, seul le maître des lieux manque. Nous trouvons ici la parfaite illustration de la volonté d'Haring de mettre à la portée de tous les budgets son art (ou des objets qui en sont dérivés en tout cas), le merchandising devient un médium pour lui. De plus, quoi de mieux que ce type de boutique au caractère unique pour assurer son autopromotion. Ajoutons que le plus grand soutien pour ce projet venait d'Andy Warhol: les deux hommes partageaient une propension à franchir les frontières séparant art et domaines plus pragmatiques (commerce, publicité, quotidien). Tracey Emin et Sarah Lucas avaient également compris l'intérêt de l'autopromotion au sein de leur Shop. Sauf que l'exemple britannique reste nettement moins développé que cette reconstitution du Pop Shop.

7 La salle dédiée à Martin Kippenberger, Candidature à ma rétrospective ${ }^{8}$, met au centre du débat le statut de l'artiste entrepreneur, entouré de petites mains et tordant le cou à l'image de l'artiste solitaire. Les salles 9 et 10, interdites aux mineurs, évoquent l'autopromotion et la construction d'une image médiatique au travers de la confusion entre l'art et la vie. L'exemple le plus flagrant reste celui de Koons et de Made In Heaven, le mettant en scène avec sa femme de l'époque, La Cicciolina. Utilisant la publicité comme point de départ ${ }^{9}$, Made In Heaven a été décliné avant et après le mariage de Koons avec la politicienne-porno star. Et dans Made In Heaven comme dans ses variations, il s'agit autant de promouvoir l'artiste-même que son œuvre. Le côté sulfureux et la confusion entre mise en scène et vie maritale n'en feront que davantage 
pour la notoriété de Koons, tout du moins pour ce qui est des tabloïds... La perte de repères est également de mise avec les travaux de Cosey Fanni Tutti, Magazine Actions. A la fois artiste et modèle dans des magazines pornographiques, le spectateur ne sait plus s'il s'agit de l'artiste qui présente un travail ou de la playmate qui expose son gagnepain. L'autoportrait et l'exposition médiatique sont d'ailleurs un des thèmes chez Piotr Ulánski (notamment dans Polesploitation, où l'artiste fait figurer, entre autres, un cliché où il figure, paru dans le magazine Vogue).

Les Young British Artists feraient presque figure d'artistes «calmes » au milieu de la nébuleuse de photographies à caractère pornographique et de la vidéo d'Andréa Fraser la mettant en scène en train de coucher avec un collectionneur dans une chambre d'hôtel, dans le but de réaliser une œuvre.

Le spectre de Warhol n'est jamais très loin chez les YBAs. Dans l'œuvre Pop, Gavin Turk se représente prenant la pose adoptée par Elvis Priestley dans les œuvres de Warhol, mais le fait sous les traits de Sid Vicious ${ }^{10}$. Il réutilise et détourne la plaque bleue de l'English Heritage apposant son nom dessus, se faisant passer lui-même à la postérité. Et alors que Ingo, Torsen et ses jumeaux assis chacun sur une chaise, ce qui a de quoi surprendre le visiteur, met en exergue les idéaux de production en série chers à Warhol, la salle Beautiful Inside My Head et ses œuvres d'or et de diamants sont un écho parfait aux Gems de Warhol.

10 «Pop Life» se termine sur l'espace consacré à Takashi Murakami. Ici beaucoup d'œuvres marquées par une collaboration (avec le réalisateur McG et l'actrice Kirsten Dunst pour un clip vidéo, ou avec la marque Louis Vuitton). Oeuvres qui, avant d'être exposées dans un musée, avaient davantage leur place dans des magasins.

11 Murakami développe les partenariats au travers de son entreprise Kaikai Kiki Co., créée au début des années 90. Grâce à celle-ci, le modèle de la Factory de Warhol est déclinée, développée. La théorie du "Superflat » met sur le même plan culture élitiste et culture populaire, leur donnant une importance égale dans son travail. Et Murakami s'affiche avec créateurs et célébrités, comme son illustre prédécesseur, d'ailleurs. La boucle est bouclée.

\section{NOTES}

1. Andy Warhol \& Pat Hackett, Popism: The Warhol'60s, New York, 1980, p.108.

2. L'exposition sera présentée du 6 février au 9 mai 2010 à la Hamburger Kunsthalle puis à la National Gallery of Canada, Ottawa du 11 juin au 19 septembre 2010.

3. Waldemar Januszczak, « Pop Life Opens at the Tate Modern », The Sunday Times, 4 octobre 2009, http://entertainment.timesonline.co.uk/tol/arts_and_entertainment/visual_arts/ article6855190.ece, 11/01/10.

4. Il s'agirait de Damien Hirst, selon Matthew Collings, « What I Thought After I Came Out of 'Pop Life' ", Artinfo, $1^{\text {er }}$ décembre 2009, http://www.artinfo.com/news/story/33206/what-i-thoughtafter-i-came-out-of-pop-life/, 14/01/10. 
5. Les Shokugan sont des figurines qui sont offertes dans des paquets de chewing-gums ou de bonbons, financièrement à la portée de tous.

6. "Pop Life ", Jack Bankowsky, dans le catalogue Pop Life, Art in a Material World, dirigé par Jack Bankowsky, Alison M. Gingeras et Catherine Wood, Tate Publishing, Londres, 2009, p.31.

7. Peter Nagy est celui qui est à l'origine de la renaissance de la galerie itinérante et expérimentale «Nature Morte» alors que Meyer Vaisman est le co-propriétaire de la galerie International with Monument.

8. Du nom de l'exposition de Kippenberger qui a eu lieu au Centre Pompidou en 1993.

9. Made In Heaven a d'abord été un panneau publicitaire géant commandé par le Whitney Museum à l'occasion de l'exposition Image World, établissant le lien entre arts et médias.

10. Bassiste des Sex Pistols.

INDEX

Thèmes : Trans'Arts

\section{AUTEUR}

\section{CLÉMENCE SIMON}

Université Paris VII 